\title{
INVESTIGATION OF THE ARC CHARACTERISTICS OF SWITCHING DC ARCS ON HYDROGEN CONTAINING GAS MIXTURES
}

\author{
D. Gonzalez ${ }^{a *}$, S. Gortschakow ${ }^{a}$, S. Yu ${ }^{b}$, F. Werner ${ }^{b}$ \\ ${ }^{a}$ INP Greifswald, Felix-Hausdorff-Str. 2, 17489 Greifswald, Germany \\ ${ }^{b}$ TDK Electronics, Gradestraße 50, 12347 Berlin, Germany \\ * diego.gonzalez@inp-greifswald.de
}

\begin{abstract}
The characteristics of switching DC current arcs in hydrogen containing gas mixtures under pressure were investigated using a model chamber. The switching device consists of an electro-mechanic double breaker unit with copper contacts. High-speed imaging and spectroscopy were used to observe and to characterize the switching arc. The experiments indicate how the dynamic interaction of an external magnetic field with a high-pressure discharge causes an elongation and twisting of the arc-channel and consequently a voltage increase. Comparative measurements with and without external magnetic field indicate a strong influence of this factor. $\mathrm{Cu}$ and $\mathrm{N}$ lines together with strongly broadened $\mathrm{H}$ lines were observed.
\end{abstract}

Keywords: arc behaviour, external magnetic fields, gas filled contactors, hydrogen, overpressure.

\section{Introduction}

The increasing use of hybrid cars and more recently the growing market of pure electric cars with batteries of also increasing capacity requires the use of switching devices, contactors, of also increased current interrupting capability.

Recent results about DC- switching devices under $\mathrm{H}_{2} / \mathrm{N}_{2}$ like by Shiba, Kaneko and co-workers [1], which studied the breakdown characteristics of the $\mathrm{H}_{2} / \mathrm{N}_{2}$ gas mixture as well as its influence on the interrupting performance of relays, have shown that compared to air $\mathrm{H}_{2} / \mathrm{N}_{2}$ at 1 bar possess a higher electric strength and permits to obtain shorter arcing times. Also the combination with magnetic arc blowing allows for further reduction of arcing time. According to their results the mixture proportion of $80 \%$ for $\mathrm{H}_{2}$ and $20 \%$ for $\mathrm{N}_{2}$ appeared as the most practical. Sawa, Suzuki and co-workers [2] presented results comparing the influence of gas $\left(\mathrm{H}_{2}, \mathrm{~N}_{2}\right.$, He and air) and working pressures ( 0.5 bar to 4 bar) on the arc voltage and current characteristics as well as arcing time for $100 \mathrm{~V}$ and 30 A DC relays. These authors found that the arcing time was shorter using $\mathrm{H}_{2}$ followed by $\mathrm{N}_{2}$, air and $\mathrm{He}$, which shows up to 7 time larger arcing times than $\mathrm{H}_{2}$. Also the arc duration was inversely proportional to working pressure. This effect was more clear by arc currents over $20 \mathrm{~A}$ and independently of filling gas. Regarding contact resistance, its increase by switching on air was of about factor two compared to the other gases, which is a consequence of oxidation processes on air. Schrank, Gerdinand and co-workers [3] presented results regarding the advantages of magnetic field blowing for DC air circuit breakers (operating voltages around $330 \mathrm{~V} \mathrm{DC}$ ). Also results of Volm [4] indicate improvements of arc switching performance for $1000 \mathrm{~V}$ and $40 \mathrm{~A}$ air relays by means of magnetic blowing combined with outgassing from polymer components.

The main interest of our investigation was to observe and subsequently to characterize the arc inside gas filled contactors (GFC). To avoid the formation of explosive atmospheres containing hydrogen are GFC's manufactured gas-tight, making difficult the optical access to the arc chamber, which is made of non transparent materials like Aluminum-oxide ceramics and furthermore enclosed in polymer casings. For this reason we decide instead of modify a real contactor, to recreate the geometry of its chamber using a transparent material like polymethyl methacrylate (PMMA) but keeping the mechanics and other components of the real device. The constructed GFC-model allows for optical observation of the arcing volume, making therefore possible to observe the arc dynamics, to collect its radiation and to measure the dynamic pressure change during arcing.

\section{Experimental methods}

\subsection{Experimental setup}

An outline of the experimental setup and of the GFCmodel is presented in Fig. 1. It consists of a double bridge contactor with a $12 \mathrm{~V}$ coil. The fixed contacts and moving bridge are made of copper. The PMMA chamber has an internal volume of around $20 \mathrm{~cm}^{3}$. The filling gas pressure can be varied from $1 \ldots 7$ bar. Although different gas mixtures can be used, our investigation focused on $\mathrm{H}_{2} / \mathrm{N}_{2}$ in a $80 \% / 20 \%$ mixture (purity of 5.0).

\subsection{Current delivery}

For the delivering of the arc current a CLR-circuit with 10 CL-groups in a transmission-line like configuration was used [5]. The resulting rectangular-shaped 


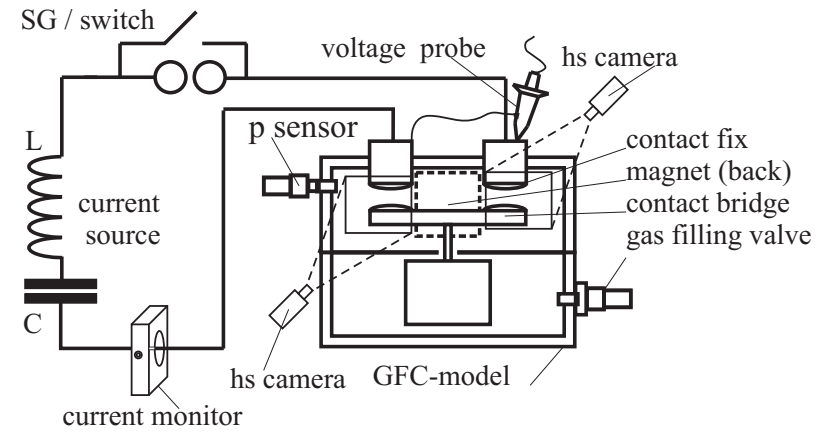

Figure 1. Experimental setup for the investigation of switching arcs in DC gas filled contactors.

current-pulse has a duration of $28 \mathrm{~ms}$. The charging voltage for the different experiments series was varied between 500 and up to $1000 \mathrm{~V}$. The amplitude of the current was of 80 and up to $160 \mathrm{~A}$ respectively. Due to the relative high effective inductivity of the circuit $(64 \mathrm{mH})$ the delivered currents show a near impressed nature.

\subsection{Methods}

The arc current and voltage signals were measured using a Pearson 1423 current monitor and a Tektronix 6015A voltage probe respectively. The current of the contactor's coil was detected using a Tektronix TCP202 Hall current probe. For the dynamic measurement of pressure during arcing a Piezosensor model PCB-105C was used. The electrical signals were recorded with a Tektronix oscilloscope model DPO 4054. The arc images were recorded using two high-speed cameras of the series Y4 and Y6 from Integrated Design Tools. The arc spectrum was determined with a compact spectrometer with a temporal resolution of $1-2 \mathrm{~ms}$ (Avantes VIS-spectrometer, AvaSpec-ULS2048, wavelength 300 to $1000 \mathrm{~nm}$ ). The fiber optic of the spectrometer was covered by a cosinus difussor to reduce light intensity. It was oriented over the same line of view of the cameras. The spectrometer was calibrated by means of a tungsten strip lamp (OSRAM Wi 17/G) in units of spectral radiance. Metal Interference Filters (MIF) for the $\mathrm{H}_{\alpha}$ line at $656 \mathrm{~nm}$ and for the $\mathrm{Cu}$-line at $525 \mathrm{~nm}$ with a full width at half maximum - FWHM of about $10 \mathrm{~nm}$ were placed before the lens of the high-speed cameras.

Values between 4 and up to 7 bar were used for filling pressure during a given experiment series. Before filling the chamber was evacuated down to 150 mbar by a membrane pump. Different experiments to determine the arc dynamics, pressure change during switching and the influence of filling pressure on arcing time and arc appearance were carried out.

\section{Results and discussion}

\subsection{Characteristics of the switching arc}

The characteristics of the switching arc are shown in Fig. 2. The images of the high speed camera in- dicate how the arc will be elongated and twisted, which explains the observed strong voltage fluctuations. According to results of Shea [6] and Rieder [7] the influence of magnetic fields should cause, besides the elongation of the arc through Lorentz-forces, also an increase of thermal losses because of the created turbulent cooling. This leads to a reduction of arc temperature and consequently of plasma conductivity, hence the additional increase on arc voltage.

\subsection{Influence of external magnetic field on arc-behavior and voltage characteristics}

Fig. 3 shows the typical characteristic of the arc current and voltage signals as well as dynamic pressure during a switching operation with the GFC-model. The considered time for the definition of arcing time and arc-extinction time are also depicted in Fig. 3. The arc-extinction time represents the interval where the arc is effectively elongated as a result of the combined effects of Lorentz-forces and turbulent convective gas flow inside the arc chamber. The dynamic characteristic is noticed on the abrupt changes of voltage value, depending on the effective change of plasma conductivity and arc-length. Also the observed pressure increase corresponds to the beginning of the arc-extinction time indicating the start of the noticeable influence of magnetic field and filling pressure on the thermal characteristic of the arc plasma. The arc-extinction time ends logically when the current is effectively driven to zero. After the effective switching, the pressure decays slowly because of the thermal delay of the heated filling gas. Also the rest voltage of the current source capacitors stays on the GFC-model contacs a longer time.

The recordings of Fig. 2 and Fig. 3 regarding arcvoltage indicate a strong fluctuating behaviour, where its value can change very quickly from some hundreds to thousand of volts. This is due to the combined effects of arc-elongation and resulting turbulent deflection through the interaction of arc-current and external magnetic field. To estimate this influence we choose to use the equivalent arc-voltage vs. current characteristic, plotting the temporally associated values of current and arc-voltage from several measurements (Fig. 4). Comparing the considered cases it is evident that a external magnetic field causes an additional voltage increase and therefore a more efficient current limiting. Although the depicted linear regression curves of the gathered data in Fig. 4 show a relative small difference, it is important to remark that with external magnetic fields the fluctuations are stronger, both in frequency and magnitude and in particular when the current droops below $20 \mathrm{~A}$.

\subsection{Influence of filling pressure on arcing time}

According to results of [2] the filling pressure influences considerably the arc extinction characteristics. The results of our experiments regarding this factor are 

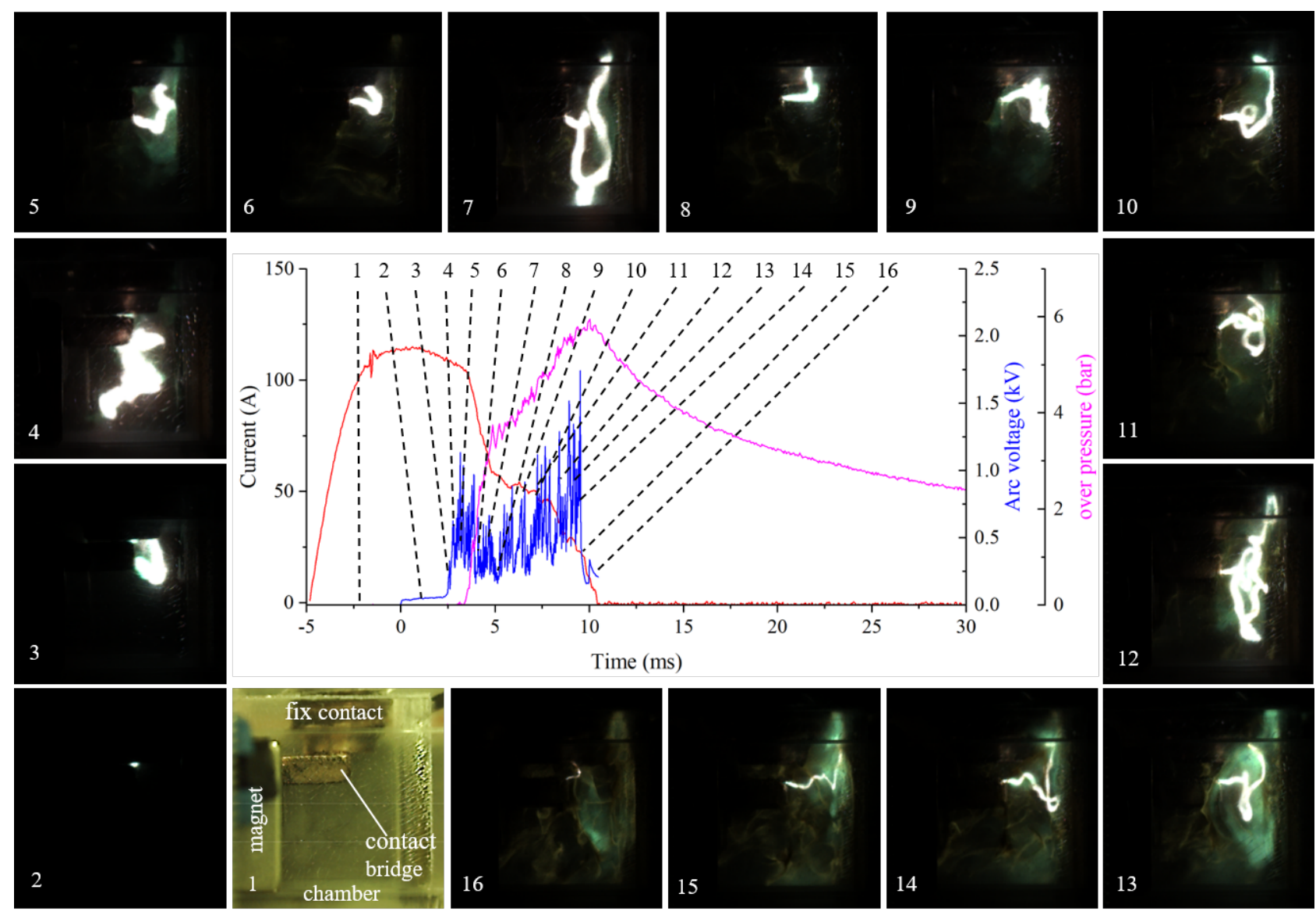

Figure 2. High-speed images of the switching arc, right side of the GFC-model, and corresponding time sequence on the arc-voltage, current and pressure waves. Frame rate of Y6 camera: $4000 \mathrm{fps}$; Exposure time: $1 \mu \mathrm{s}$

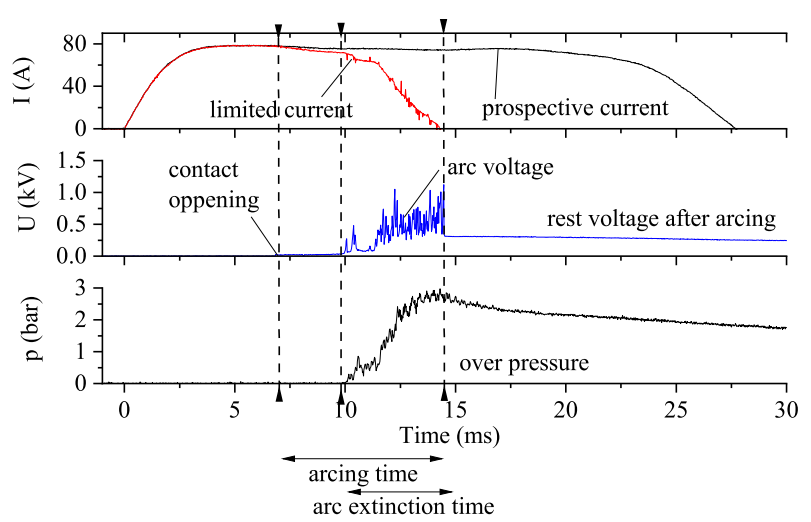

Figure 3. current, arc-voltage and over-pressure of a typical switching operation of the GFC-model.

depicted in Fig. 5. Although arcing time and arc extinction time appear to be independent on the filling pressure, Fig. 5 shows a slight reduction of the data scattering, in particular by 7 bar the arc-extinction time is notably reduced compared to that of pressures between 4 and 6 bar. Although the considered pressure interval is small, this result points to the influence of filling gas and pressure on the switching behavior, which depends rather on the burning conditions for the arc than on the switching device mechanics. The registered times for the $80 \mathrm{~A}$ are nevertheless around

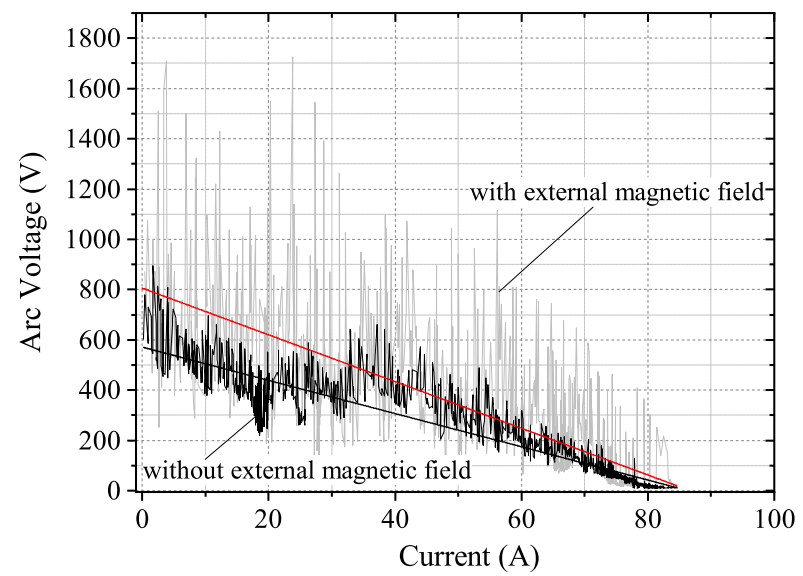

Figure 4. Influence of the external magnetic field on the arc-voltage vs. current characteristic for the GFCmodel.

2.5 times higher than those reported by [2]. This is most probably due to the differences between used current-sources, since for [2] the voltage was of $100 \mathrm{~V}$ and as it appears, the circuit was of purely ohmic nature. The tendency of reduction of arc extinction time as a function of filling pressure reported there was not clearly observed during our measurements, which is probably due to some kind of saturation 


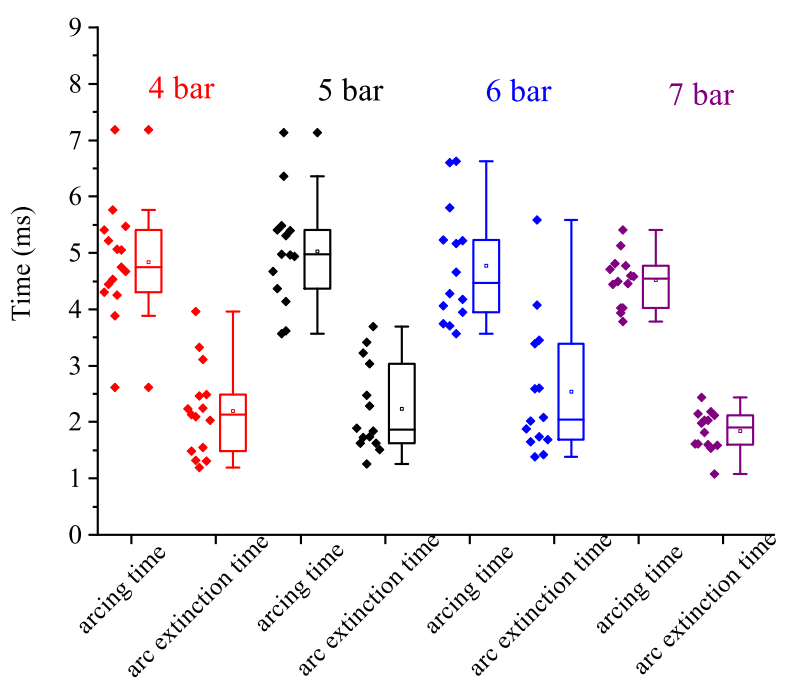

Figure 5. Influence of the filling pressure on the total arcing time and an the total arc extinction time for the GFC-model.

effect because of the geometric characteristics and components of the GFC-model.

\subsection{Characteristics of the arc spectrum}

In a first attempt to characterize the thermal properties of the arc plasma inside the GFC-model, qualitative spectroscopic measurements were carried out. The results are shown in Fig. 6. One of the difficulties presented here was the unstable behavior of the arc inside the chamber (see Fig. 2), preventing a spatially resolved measurement of its spectrum. It was nevertheless characterized by a strong broadened $\mathrm{H}_{\alpha}$ line. Further measurements overriding the intensity of the $\mathrm{H}_{\alpha}$ permit to identify other species like $\mathrm{H}_{\beta}$ and $\mathrm{H}_{\gamma}$ lines as well as copper and nitrogen lines. Also carbon and oxygen lines could be identified, which should result because of arc ablative effects on polymer parts of the mechanics and on the PMMA chamber. Although it is not yet possible to quantify the plasma characteristics from the obtained spectrum, the absence of ion-lines is an indication that the arc temperature is probably under $12000 \mathrm{~K}$.

\section{Summary}

The characteristics of DC switching arcs in gas filled contactors was investigated with a experimental model using a PMMA chamber. The results indicate how the arc will be elongated and twisted because of the combined effects of external magnetic field and turbulent cooling in the high pressure chamber. The consequence is a remarkable increase of arc-voltage though accompanied by strong fluctuations. The final effect is however an effective improvement of the current limiting performance of the device. External magnetic fields allow also to reduce the effective switching time and therefore the arcing stress on the contactor. An

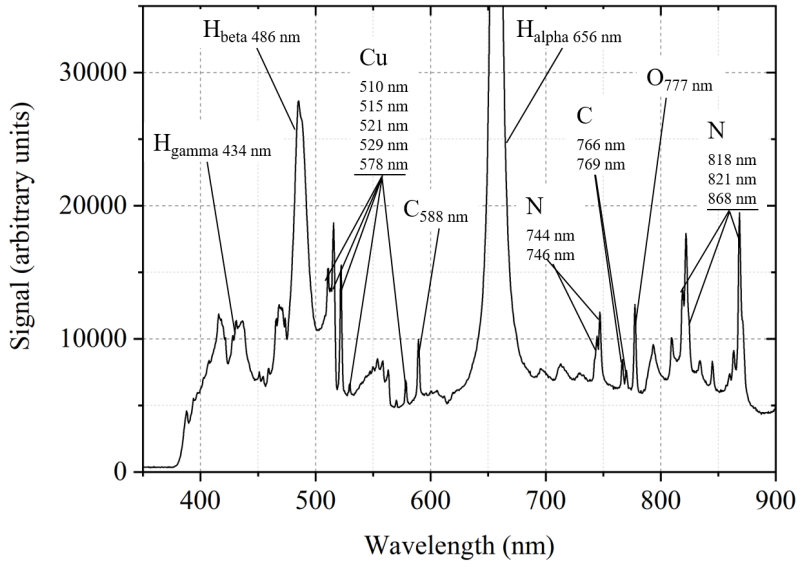

Figure 6. Emision spectrum of the switching arc inside of the GFC-model.

increase on filling pressure appears to influence the resulting arc-extinction time scattering. Its mean value was though similar within the considered pressure interval between 4 and 7 bar. The emission spectrum shows predominantly atomic lines. In particular a strong broadened $\mathrm{H}_{\alpha}$ dominates the collected radiation intensity. Also copper and nitrogen lines as well as carbon and oxygen lines, probably because of arc ablative processes on polymer components and on the arc chamber (PMMA), were observed. Further experiments regarding the thermal characteristics of the arc plasma are in preparation.

\section{References}

[1] Y. Shiba et al. Study of DC Circuit breaker of $\mathrm{H}_{2}-\mathrm{N}_{2}$ Gas Mixture for High Voltage. Electrical Engineering in Japan., 174(2):1407-1413, 2011. doi:10.1002/eej. 21042.

[2] K. Sawa et al. Influence of sealed gas and its pressure on arc discharge in electromagnetic contactor. IEEE Holm Conference on Electrical Contacts, pages 236-241, 2017. doi:10.1109/HOLM. 2017.8088093.

[3] T. Schrank et al. Breaking performance of a circuit breaker influenced by a permanent magnetic field at DC voltages up to $450 \mathrm{~V}$. Proceedings of the ICEC-ICREPE, pages 236-241, 2012. doi:10.1049/cp.2012.0618.

[4] D. Volm and F. Winkler. Development of a compact relay for high voltage switching of up to $1000 \mathrm{~V}$ and $40 \mathrm{~A}$. International Conference on Electrical Contacts, 27:144-148, 2014.

[5] A. Khakpour et al. Impact of different vacuum interrupter properties on high-current anode phenomena. IEEE Transactions on Plasma Science, 44:3337-3345, 2018. doi:10.1109/TPS. 2016. 2625862.

[6] J. Shea, E. Heckman, and J. Suarez Guevara. DC Arc Properties in a DC Magnetic Fields. 64th IEEE Holm Conference on Electrical Contacts/29th International Conference on Electrical Contacts, 64(1):195-202, 2017. doi:10.1109/HOLM. 2018.8611725.

[7] W. Rieder and A. Eidinger. Das Verhalten des Lichtbogens im transversalen Magnetfeld (Magnetische Blasung). Archiv für Elektrotechnik, 43(2):94-114, 1957. doi:10.1007/BF01479337. 